\title{
TOWARDS SERIES MEASUREMENTS OF THE LHC SUPERCONDUCTING DIPOLE MAGNETS
}

\author{
L. Walckiers, Z. Ang, J. Billan, L. Bottura, A. Siemko, P. Sievers, R. Wolf, \\ CERN, 1211 Geneva 23, Switzerland
}

\section{Abstract}

Extensive power tests of the LHC dipole magnets required the development of new techniques to study the quench and training behaviour. Magnetic measurements of short and long model dipoles have allowed to understand and quantify the time dependent behaviour of the field quality during the current flat top needed during beam injection. The experience gained is employed for the design of the measuring tools presently under construction for the series measurements of the LHC dipole magnets. The economically important issue of how many magnets have to be measured in the superconducting state is addressed in view of the field quality required for the performance of the LHC.

\section{INTRODUCTION}

The experience from previous accelerators based on superconducting magnets stresses the difficulty to control the beam during injection and beginning of the acceleration due to time dependent effects of the sextupole component of the field. Furthermore studies of the dynamic aperture of the LHC accelerator [1] pointed to stringent specifications for higher harmonics. The main dipole field is only $0.54 \mathrm{~T}$ at injection for a nominal field of $8.3 \mathrm{~T}$ at collision energy.

Seven $10 \mathrm{~m}$ long twin aperture magnets based on the previous design and more than ten $1 \mathrm{~m}$ long models with the present structure were tested in cold conditions. The measured training characteristics and the magnetic measurements performed are analysed to give detailed arguments to specify the extend of the costly cold measurements of the series magnets.

\section{POWER TESTS}

\subsection{Training of the $1 \mathrm{~m}$ long dipole models}

Cold tests of ten $1 \mathrm{~m}$ long single and twin aperture models, thoroughly instrumented, allow to classify the training quenches of the magnets in two ranges of behaviour. Their construction is described in [2].

Some magnets show a training (less than 3 quenches) starting below or around the nominal field of $8.3 \mathrm{~T}$ and going to about $8.8 \mathrm{~T}$, corresponding to $90 \%$ of the cable limit. All these quenches start in specific weak points of the winding located either in the ends of the coils windings, or in the "splice-layer jump" region (ramp of the inner layer conductor to the outer layer and connection to the outer layer cable). These findings have allowed to improve that area in the last magnets tested.

A second part of the training, starting at the range of 8.8 $\mathrm{T}$ has about the same average slope for all magnets built with the nominal structure (Fig. 1). Although the same specific regions of the windings are generally involved, recent tests show that changes in the structure are likely to improve this behaviour: decrease the sensitivity of the cable to local energy deposition [3], lower the average compression of the winding or change the material of the collars. This regular training is again found after a thermal cycle to room temperature.

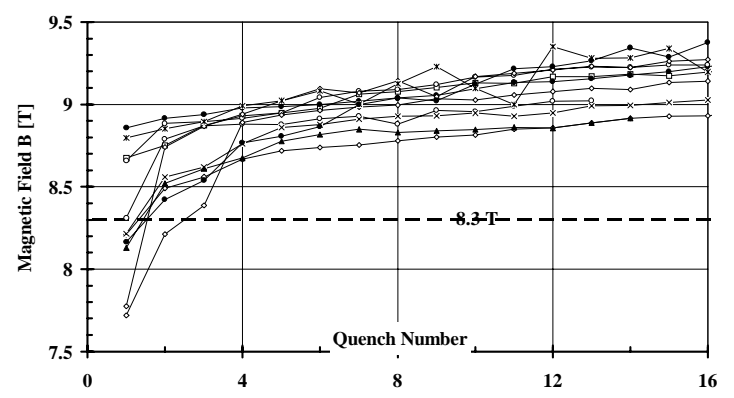

Figure 1: Training curves of the $1 \mathrm{~m}$ long model magnets tested.

\subsection{Defining the power tests for the series}

Voltage taps are the simplest tool to localise where a training quench starts in the winding. Series magnets will however not be equipped with them and even full length prototype magnets to come will have a limited number of voltage taps.

Dedicated 'Quench Antenna' coils help to determine where the training quenches start and to deduce the weak points in the magnets coils. They provide as well some information about the mechanism generating these early quenches [4]. The $15 \mathrm{~m}$ long shaft, fitted with modular coils and built for the magnetic measurements will as well serve for quench localisation (see section 4).

Since the first part of the training is of concern for magnets to be installed in the LHC, the first quench of any series magnet will be recorded with fully debugged instrumentation and in condition of temperature and current cycles as near as possible to machine conditions.

The integrity of the superconducting cable is systematically verified by a quench at $4.3 \mathrm{~K}$ after the training since its value is confidently related to the limit at superfluid temperature [5]. The RRR (ratio of the 
electrical conductivity of the copper matrix between room and low temperature) is also systematically measured because of its importance for protection of the magnets during the quenches. The sensitivity of the quench level to the current ramp rate is established.

\section{FIELD QUALITY MEASUREMENTS}

\subsection{Expected field harmonics}

The three following sources of field imperfections can degrade the performances of the beam:

- Geometric: accuracy in the positioning of the coils.

- Decay: amplitude of the decay with time at the injection plateau [6]. The snap-back at the start of the acceleration has the same value but with an opposite sign. The data of table 1 come from the measurements of 6 single aperture $1 \mathrm{~m}$ long models measured.

- Ramp: harmonics created by the coupling current between the strands of the superconducting cable for the nominal ramp of $8 \mathrm{~T}$ in $20 \mathrm{~min}$. The data are obtained from measurements of 5 apertures of the first generation $10-\mathrm{m}$ long models. They are scaled to the present magnets structure and apply for injection field.

\begin{tabular}{||c|c|c|c|c|c|c||}
\hline \hline & \multicolumn{2}{|c|}{ Geometric } & \multicolumn{2}{|c|}{ Decay } & \multicolumn{2}{c||}{ Ramp } \\
\hline & $\sigma$ & Unc. & $\sigma$ & Unc. & $\sigma$ & Unc. \\
\hline $\mathrm{a}_{2}$ & 1. & 0.3 & 0.5 & 0.2 & 1. & 0.4 \\
$\mathrm{~b}_{3}$ & 0.5 & 0.3 & 0.3 & 0.13 & 0.2 & 0.08 \\
$\mathrm{~b}_{4}$ & 0.1 & 0.05 & 0.04 & 0.015 & 0.04 & 0.02 \\
$\mathrm{a}_{4}$ & 0.1 & 0.05 & 0.13 & 0.05 & 0.11 & 0.05 \\
$\mathrm{~b}_{5}$ & 0.05 & 0.05 & 0.1 & 0.04 & 0.04 & 0.02 \\
\hline
\end{tabular}

Table 1 : Standard deviations $(\sigma)$ and uncertainties on the averages (Unc.) per octant expected for the LHC dipole magnets. The $b_{n}, a_{n}$ are the normal and skew harmonics of the field errors relative to the main field at a reference radius of $10 \mathrm{~mm}$, given in units of $10^{-4}$.

Table 1 gives the present estimation of the standard deviation of the harmonics of the field for the LHC main dipoles. The uncertainties of the averages due to possible systematic effects on a production line of magnets are also given. Magnets coming from a given manufacturer and having superconducting cables from the same production line will be assembled in the same octant of the machine. Beam stability calculations are based on the hypothesis that the uncertainties of the average per octant are equal to the expected maximum for a production line.

Values for the averages of the non-allowed harmonics indicated for the ramp and decay effects are probably pessimistic with the lack of statistics. Recent developments to increase and stabilise the interstrand resistance will lead to further improvements [7].

\subsection{Comparison with tolerances from the beam optics}

The coupling between the vertical and horizontal betatron tunes has to be controlled during the beam acceleration to within 0.003 , corresponding to 0.025 unit for the integrated $a_{2}$. Similarly a specification of a maximum excursion of 2 unit in chromaticity requests a control of the integrated sextupole, $b_{3}$, to 0.015 unit. The dynamic aperture remains satisfactory if the octants average of both octupoles terms are lower than 0.015 unit and if the control of the integrated decapole with the help of the end correctors is within 0.03 units.

\begin{tabular}{||c|c|c|c|c|c|c||}
\hline \hline & \multicolumn{2}{|c|}{ Geometric } & \multicolumn{2}{|c|}{ Decay } & \multicolumn{2}{c||}{ Ramp } \\
\hline & $\Delta / \sigma$ & $\Delta /$ Unc. & $\Delta / \sigma$ & $\Delta /$ Unc. & $\Delta / \sigma$ & $\Delta /$ Unc. \\
\hline $\mathrm{a}_{2}$ & 0.03 & 0.08 & 0.05 & & 0.03 & \\
$\mathrm{~b}_{3}$ & 0.03 & 0.05 & 0.05 & 0.1 & 0.08 & 0.2 \\
$\mathrm{~b}_{4}$ & 0.15 & 0.3 & 0.4 & & 0.4 & \\
$\mathrm{a}_{4}$ & 0.15 & 0.3 & 0.1 & & 0.14 & \\
$\mathrm{~b}_{5}$ & 0.6 & 0.6 & 0.3 & 0.5 & 0.8 & 1 \\
\hline \hline
\end{tabular}

Table 2 : Tolerances specified for beam stability $(\Delta)$ divided by the standard deviations $(\sigma)$ or uncertainties of the averages (Unc.) per octant.

Table 2 indicates the relative accuracy to which the standard deviation and the average per production line should be measured for the series of the LHC dipole magnets. Measurements of the coupling and chromaticity during the beam acceleration are difficult to realise for a correction in closed loop. Tables to predict the skew quadrupole and the normal sextupole are therefore needed with the accuracy expressed in table 2. These predictions will require to measure all magnets in cold conditions unless the standard deviations of the Decay and Ramp imperfections are found to be smaller than those of Table 1 for the preseries magnets.

Some care will also be needed during the magnets production to ensure that the Decay and Ramp effects do not introduce systematic octupoles that can be detrimental for the dynamic aperture.

\subsection{Reproducibility needed for the harmonic content}

In addition to the spread and the bias over a series of magnets, the stability with respect to previous or external conditions (current history, quenches, helium bath temperature) was also studied on the existing magnets.

The reproducibility of 0.015 unit for the normal sextupole $b_{3}$ corresponds to the following variations around injection field: temperature change of the superconductor of $0.04 \mathrm{~K}$ or current overshoot in the magnet in the range of $0.1 \mathrm{~A}$. Furthermore the time previously spent at high field or below injection current influences the decay of $b_{3}$ at injection current within a range of 1 unit. We have measured that these variations are larger for long magnets than for the $1 \mathrm{~m}$ long models tested. Therefore prediction laws for magnets from 
different manufacturers or wound with cables having different properties will have to be measured with the accuracy quoted above to choose reference magnets.

The correction of the decapole term will however be much easier to control since it varies by less than 0.08 unit, taking into account all of these effects.

\section{TOOLS FOR THE SERIES MEASUREMENTS}

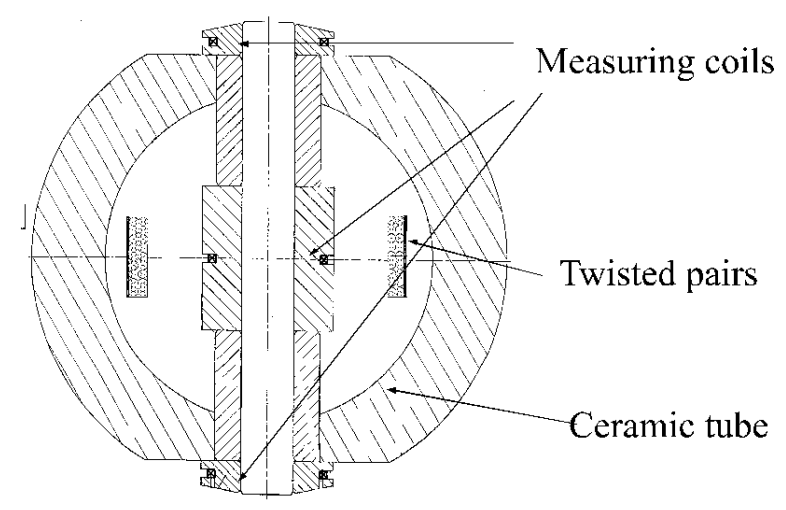

Figure 2 : Cross-section of the harmonic coils for the cold magnetic measurements and quench localisation.

Early it appeared that scanning the pair of $14.2 \mathrm{~m}$ long apertures of each dipole piece wise with a single coil would need too much time, in particular the measurement of time dependent behaviour. Based on the experience gained with long and slender ceramic shafts, driving measuring coils, an integral coil is now under construction. It consists of 13 identical modules each 1.26 $\mathrm{m}$ long and mechanically assembled into one long shaft spanning the total length of the aperture. Each module consists of a ceramic pipe, providing the necessary rigidity, onto which the tangential measuring coils are mounted (Fig. 2). Between each module a length of 110 $\mathrm{mm}$ is required to house the rotating bearings and the cable interconnects. Careful calibration and sorting of the individual coils allow to achieve a rejection of the main harmonic to better than 500 without electronic bucking. Each coil is read out individually. That requires finally 39 micro-pin connectors and strands of twisted pairs, designed and verified that noise picked up during the rotation of the shaft by residual loops of wires is negligible. Each coil within the fully assembled unit will be calibrated with respect to harmonics and orientation via a resistive reference dipole magnet. Using one long shaft in each aperture allows to measure simultaneously within about $10 \mathrm{~s}$ the harmonics along the whole magnet, very efficient for the study of dynamic effects and their local and integral behaviour. Moreover, quench detection and localisation is readily provided by these coils.

\subsection{The inactive lengths along the measuring shaft}

The length not covered by measuring coils has to be chosen as near as possible to the transposition pitch of the cable to avoid errors from the strong longitudinal periodic pattern of the field. 12 transposition pitches will therefore be missing in the measured field corresponding to about $10 \%$ of the effective length of the magnet. The transposition pitch of the LHC cable is expected to stay within 105 to $110 \mathrm{~mm}$. Some residual beating of the periodic pattern will occur with the constant $110 \mathrm{~mm}$ of hole length. Estimations based on the measured magnets give a maximum error of 0.03 units for the skew quadrupole component at injection field. The variation of the harmonics along the magnet axis, after averaging the contribution of the periodic pattern, has been measured to be small enough to guarantee an error of less than $1 \%$ on the values expected.

\section{CONCLUSION}

Power tests of the dipole magnets will be needed to prove that the weak points in the coils windings do not lead to quenches below the nominal field of the LHC machine. Sufficient statistics must be accumulated on the dynamic behaviour of the field harmonics before deciding on the amount of LHC dipoles to be tested cold. A prototype $15 \mathrm{~m}$ long measuring shaft is presently being assembled to address both of these types of measurements.

\section{REFERENCES}

[1] The Large Hadron Collider - Conceptual Design, CERN/AC/95-05,1995.

[2] N. Andreev et al., 'The $1 \mathrm{~m}$ long single aperture dipole test program for LHC', Proc. of $5^{\text {th }}$ European Particle Accelerator Conference, Sitges, June 1996.

[3] A.K. Ghosh et al., 'Minimum quench energies of Rutherford cables and single wires' IEEE Trans. Of Appl. Sup., 7, to appear, 1997.

[4] A. Siemko et al., "Power tests results of the first LHC second generation superconducting single aperture 1 $\mathrm{m}$ long dipole models', Proc. of $16^{\text {th }}$ International Cryogenic Engineering Conference, pp. 837-842, Kitakyushu, May 1996, Elsevier Science.

[5] L. Walckiers et al., 'Power tests of single and twin aperture superconducting dipole models for LHC', Proc. of $14^{\text {th }}$ Magnet Technology Conference, Tampere, June 1995.

[6] L. Bottura, L. Walckiers, R. Wolf, 'Field errors decay and snap-back in LHC model dipoles', IEEE Trans. Of Appl. Sup., 7, to appear, 1997.

[7] D. Richter et al., 'DC measurement of electrical contacts between strands in superconducting cables for the LHC magnets, IEEE Trans. Of Appl. Sup., 7, to appear, 1997. 